\title{
Direction identification in radio images of cosmic-ray air showers detected with LOPES and KASCADE
}

A. Nigl ${ }^{1}$, W. D. Apel ${ }^{2}$, J. C. Arteaga ${ }^{3}$, T. Asch ${ }^{4}$, J. Auffenberg ${ }^{5}$, F. Badea ${ }^{2}$, L. Bähren ${ }^{6}$, K. Bekk ${ }^{2}$, M. Bertaina ${ }^{7}$, P. L. Biermann ${ }^{8}$, J. Blümer ${ }^{2,3}$, H. Bozdog ${ }^{2}$, I. M. Brancus ${ }^{9}$, M. Brüggemann ${ }^{10}$, P. Buchholz ${ }^{10}$, S. Buitink ${ }^{1}$, H. Butcher ${ }^{6}$, E. Cantoni ${ }^{7}$, A. Chiavassa ${ }^{7}$, F. Cossavella ${ }^{3}$, K. Daumiller ${ }^{2}$, V. de Souza ${ }^{3}$, F. Di Pierro ${ }^{7}$, P. Doll ${ }^{2}$, R. Engel ${ }^{2}$, H. Falcke ${ }^{1,6}$, H. Gemmeke ${ }^{4}$, P. L. Ghia ${ }^{11}$, R. Glasstetter ${ }^{5}$, C. Grupen ${ }^{10}$, A. Haungs ${ }^{2}$, D. Heck ${ }^{2}$, J. R. Hörandel ${ }^{1}$, A. Horneffer ${ }^{1}$, T. Huege ${ }^{2}$, P. G. Isar ${ }^{2}$, K.-H. Kampert ${ }^{5}$, D. Kickelbick ${ }^{10}$, Y. Kolotaev ${ }^{10}$, O. Krömer ${ }^{4}$, J. Kuijpers ${ }^{1}$, S. Lafebre ${ }^{1}$, P. Łuczak $^{12}$, M. Manewald ${ }^{4}$, H. J. Mathes ${ }^{2}$, H. J. Mayer ${ }^{2}$, C. Meurer ${ }^{2}$, B. Mitrica ${ }^{9}$, C. Morello ${ }^{11}$, G. Navarra ${ }^{7}$, S. Nehls ${ }^{2}$, J. Oehlschläger ${ }^{2}$, S. Ostapchenko ${ }^{2}$, S. Over ${ }^{10}$, M. Petcu ${ }^{9}$, T. Pierog ${ }^{2}$, J. Rautenberg ${ }^{5}$, H. Rebel ${ }^{2}$, M. Roth $^{2}$, A. Saftoiu ${ }^{9}$, H. Schieler ${ }^{2}$, A. Schmidt ${ }^{4}$, F. Schröder ${ }^{2}$, O. Sima ${ }^{13}$, K. Singh ${ }^{1}$, M. Stümpert ${ }^{3}$, G. Toma ${ }^{9}$, G.C. Trinchero ${ }^{11}$, H. Ulrich ${ }^{2}$, J. van Buren ${ }^{2}$, W. Walkowiak ${ }^{10}$, A. Weindl ${ }^{2}$, J. Wochele ${ }^{2}$, J. Zabierowski ${ }^{12}$, and J. A. Zensus ${ }^{8}$

(Affiliations can be found after the references)

Received 7 December 2007 / Accepted 12 June 2008

\section{ABSTRACT}

Aims. We want to understand the emission mechanism of radio emission from air showers to determine the origin of high-energy cosmic rays Therefore, we study the geometry of the air shower radio emission measured with LOPES and search for systematic effects between the direction determined on the radio signal and the direction provided by the particle detector array KASCADE.

Methods. We produce 4D radio images on time-scales of nanoseconds using digital beam-forming. Each pixel of the image is calculated for three spatial dimensions and as a function of time. The third spatial dimension is obtained by calculating the beam focus for a range of curvature radii fitted to the signal wave front. We search this multi-dimensional parameter space for the direction of maximum coherence of the air shower radio signal and compare it to the direction provided by KASCADE.

Results. The maximum radio emission of air showers is obtained for curvature radii being larger than $3 \mathrm{~km}$. We find that the direction of the emission maximum can change when optimizing the curvature radius. This dependence dominates the statistical uncertainty for the direction determination with LOPES. Furthermore, we find a tentative increase of the curvature radius to lower elevations, where the air showers pass through a larger atmospheric depth. The distribution of the offsets between the directions of both experiments is found to decrease linearly with increasing signal-to-noise ratio. Significantly increased offsets and enhanced signal strengths are found in events which were modified by strong electric fields in thunderstorm clouds.

Conclusions. We conclude that the angular resolution of LOPES is sufficient to determine the direction which maximizes the observed electric field amplitude. However, the statistical uncertainty of the directions is not determined by the resolution of LOPES, but by the uncertainty of the curvature radius. We do not find any systematic deviation between the directions determined from the radio signal and from the detected particles. This result places a strong supportive argument for the use of the radio technique to study the origin of high-energy cosmic rays.

Key words. acceleration of particles - elementary particles - radiation mechanisms: non-thermal - instrumentation: detectors methods: observational - techniques: image processing

\section{Introduction}

Cosmic rays are particles penetrating the Earth's atmosphere with energies from $\mathrm{GeV}$ up to hundreds of EeVs. These particles (mostly protons) have been measured directly up to energies of $\mathrm{PeV}$. Most cosmic rays up to $\mathrm{PeV}$ energies are thought to be accelerated in our Galaxy by supernova remnants. However, the origin of the particles beyond those energies is still unclear.

Therefore, the study of high-energy cosmic rays is important to find their sources and to understand how they were accelerated. However, the flux drops with a steep spectrum from about one particle per year per square meter at PeV energies, to about one particle per century per square kilometer at energies of a hundred EeV. Because of this low flux and a certain interaction in the Earth's atmosphere, cosmic rays with energies beyond about a hundred $\mathrm{PeV}$ are measured indirectly by a cascade of secondary particles. Billions of secondary particles are produced in these cascades called extensive air showers and hundreds of thousands of them reach particle detectors on the ground.

The relative arrival times of these particles allow to determine the direction from which the primary particle entered the Earth's atmosphere. However, the identification of the source is hampered by the deflection of the primary particle by the magnetic field distribution in our Galaxy. The higher the energy, the smaller the deflection of the particle; and for very high energies larger than $10^{19} \mathrm{eV}(10 \mathrm{EeV})$, the proton cyclotron radius becomes larger than the dimension of our Galaxy so that its trajectory possibly points back to its source.

Measurement of these cosmic rays over long periods can reveal a clustering of events, which would enable the identification of their sources and the determination of the particle acceleration process (Auger Collaboration: Abraham et al. 2007).

Cosmic-ray air showers and their origin have been studied with particle detectors since Auger et al. (1939). The position 
of the shower axis on the ground is determined from the lateral distribution of particles (Mayer 1992), which decreases with distance from the shower core; and the direction is determined on the particle arrival times (Mayer 1993).

Falcke et al. (2005) and Ardouin et al. (2005) showed that radio emission from cosmic-ray air showers can also be used to determine the energy of the primary particle. This result revived the detection of air showers in the radio regime, which were first observed in 1964 by Jelley et al. (1965) and Allan \& Jones (1966). The radio emission in particle cascades is produced by electrons and positrons being deflected in the Earth's magnetic field. Both emit beamed coherent radiation called geosynchrotron emission (Falcke \& Gorham 2003). Since the electromagnetic emission is not attenuated by collisions, it reaches the ground and therefore the longitudinal shower development can be studied by observing the emission at several distances from the shower axis. The peak voltage of the radio emission detectable on the ground depends approximately linearly on the energy of the primary particle (Huege \& Falcke 2005). In addition, the radio emission increases with increasing geomagnetic angle between the cosmicray trajectory and the Earth's magnetic field (Falcke et al. 2005).

A new generation of radio telescopes like LOFAR and LOPES can digitize the signals from cosmic-ray air showers detected by many simple dipole antennae providing them with high time, frequency, and spatial resolution. These powerful interferometers can be used to determine the direction of origin of the beamed and coherent emission produced in air showers.

In this article, we present the determination of the direction of several hundreds of cosmic-ray air showers measured in the radio regime with the LOFAR Prototype Station (LOPES, Horneffer et al. 2004) at energies above $10^{16} \mathrm{eV}$. We determine the exact direction of maximum emission by searching a small 4D radio map in the direction provided by the triggering KArlsruhe Shower Core and Array particle DEtector (KASCADE, Antoni et al. 2003). The resulting distance between the radio-direction and the particle-direction is analyzed for systematic effects.

The data acquisition and event selection is described in Sect. 2. The direction determination is explained in Sect. 3. The dependencies of the shower emission maximum are elaborated in Sect. 3.3, and the results are discussed in Sect. 4.

\section{Data acquisition}

LOPES consists of 30 inverted-V-shaped dipoles placed between the particle detector stations of the KASCADE experiment. LOPES receives a coincidence trigger from KASCADE and stores $0.4 \mathrm{~ms}$ of radio data before and after its reception. The signal of each antenna is amplified, filtered in the band from $40 \mathrm{MHz}$ to $80 \mathrm{MHz}$, digitized with 80 Megasamples per second, and written to disk. The digitally stored time-series for each antenna is processed off-line, where we perform absolute calibration, we reduce radio frequency interference (RFI) by downweighting of narrow-band lines in frequency, and form a beam by adding up or correlating the time-shifted antennae for the direction given by KASCADE. In addition to the direction information, KASCADE provides parameters such as the shower core position, electron number and muon number allowing the estimation of the primary energy. More details on the LOPES data acquisition and data processing can be found in Nigl et al. (2008).

The shower direction is reconstructed by KASCADE with an accuracy of about 0.1 degrees at the zenith and the shower core position with an accuracy of about $1 \mathrm{~m}$ (Antoni et al. 2003), both for an event with an electron number of $10^{6.5}\left(\sim 10^{16.5} \mathrm{eV}\right)$. The directional accuracy improves with increasing shower size and reduces with inclination angle. Beyond 42 degrees zenith angle, the directional uncertainty is strongly increased due to the reduced acceptance of the particle detectors. The mentioned accuracy of KASCADE is valid for showers hitting the particle detector array within a centered circle with a radius of $91 \mathrm{~m}$, so that the core is at least $10 \mathrm{~m}$ inside the array.

For this work, 664 LOPES events were pre-selected with a lower limit on the muon number of $10^{5.2}\left(>10^{16} \mathrm{eV}\right)$ and a cut on the distance of the shower core from the array centre of $91 \mathrm{~m}$. These events have been acquired from 2005-11-16 to 2006-07-23.

\section{Direction determination}

The phasing of an array by digital beam-forming is usually done in direction of a source at infinite distance. However, the air shower radio emission is produced in the Earth's troposphere by an extended emission-region and therefore, the wavefront is not flat. As a first approximation, a spherical wavefront was assumed for the radio emission reaching the antennae of LOPES. The spherical wavefront is defined by a specific curvature radius (beam focus) and a shower core position on the ground. The resulting distance of the beam focus does not necessarily coincide with the distance of the maximum emission in the air shower. This needs to be investigated in shower simulations and dedicated measurements in order to study the real shape of the shower front and its relation to the position of the maximum radio emission and the position of the maximum number of particles, respectively. The relation between the maximum in the radio shower and in the particle shower might enable the determination of the particle species from the shower radio emission only. This topic is not part of this work, however Monte Carlo simulations by Huege \& Falcke (2005, Fig. 5) show an indication of a more complex shower wavefront than a spherical one.

\subsection{Four-dimensional radio image}

For each cosmic-ray event, all pixels of a four-dimensional radio image were calculated spanning azimuth, elevation, curvature radius, and time. The image was calculated with an extension of $50 \times 50$ pixels, with an angular resolution of $0.2^{\circ}$. The map was centered on the direction given by KASCADE.

The shower core position for the spherical beam-forming was taken from KASCADE. The curvature radius for the beamforming algorithm was chosen to range from $1 \mathrm{~km}$ to $10 \mathrm{~km}$ in 37 steps of $250 \mathrm{~m}$. The time resolution was the LOPES sample-time bin of $12.5 \mathrm{~ns}$. The time window was chosen to be $1.6 \mu$ s (128 samples), so much larger than the length of a typical cosmic-ray radio pulse of a few samples in time, to avoid distortion of the pulse in the image due to edge-effects in the beam-forming process. The signal has been integrated over the LOPES band from $43 \mathrm{MHz}$ to $74 \mathrm{MHz}$. The shower core position, which is used for the beam-forming process remains constant at the value provided by KASCADE. The intensity of the cosmic-ray event in the image corresponds to the power measured in the LOPES beam. The imager performs beam-forming by adding up the antennae and not by correlating them, as it is used for calculating the E-field amplitude at the position of the maximum from the image.

Oscillations in the signal shorter than the inverse of the bandwidth are not real but they are caused by over-sampling a 
bandpass broadened signal. Therefore, the final 4D image was Hanning-smoothed in time by a weighted average of every pixel with its two neighbouring pixels.

\subsection{Emission maximum}

The emission maximum in the 4D image was located, firstly, by determining the time of maximum emission for each slice in curvature radius by selecting the 2D-azimuth/elevation-slice containing the pixel with the maximum intensity. For this search in time, 41 of the 128 available samples around the window center were scanned. Secondly, each of the 2D curvatureslices at the temporal maximum is searched for the position of the brightest point source (FINDPOINTSOURCES function of CASA/AIPS++, www . aips2.nrao.edu, v1.9, build 1360, Shannon 1996). A plot of an example event can be found in Fig. 4. In case multiple sources were found, the direction of the strongest one was saved and the other sources were classified as side-lobes. As a result, the position in time and the direction in azimuth-elevation were calculated as a function of curvature radius.

The two-dimensional spatial shape of the cosmic-ray radio emission in the image is mainly determined by the beam-shape (point spread function, PSF) and depends on the layout of the selected antennae. The PSF of the emission is assumed to be spherical at the zenith. At lower elevations, the resolution in elevation of LOPES is reduced by the sine of the elevation angle. A theoretical uncertainty for the determination of the direction is obtained following Taylor et al. (1999):

$\Delta \alpha_{\min }= \pm \frac{1}{2} \frac{\epsilon_{\mathrm{N}}}{\epsilon_{\mathrm{P}}} \frac{\lambda}{D \cos \theta}$.

Here $\Delta \alpha_{\min }$ is the minimum statistical uncertainty of the determined direction, $\epsilon_{\mathrm{P}}$ is the electric field strength of the detected pulse, $\epsilon_{\mathrm{N}}$ is the noise in the LOPES beam, $\lambda$ is the wavelength, $D$ is the diameter of the LOPES antenna array, and $\theta$ is the zenith angle. The angular resolution $\vartheta=\lambda / D \cos \theta$ or beam width of LOPES is $2.2^{\circ}$ for the zenith $(\lambda=5 \mathrm{~m}, D \cos \theta=130 \mathrm{~m})$. An example-event of LOPES at an estimated primary energy of $50 \mathrm{PeV}$, an electron number of $10^{6.7}$, and a zenith angle of $13.0^{\circ}$ (for LOPES and KASCADE) was obtained with a E-field peak signal-to-noise ratio $(\mathrm{SNR})$ of $\epsilon_{\mathrm{P}} / \epsilon_{\mathrm{N}}=8.5$ resulting in an uncertainty of $0.13^{\circ}$, which is about the same accuracy as mentioned for KASCADE.

\subsection{Dependencies of the measured electric field}

In the time domain, the air shower pulse is expected at approximately $1.8 \mu$ s before the trigger from KASCADE arrives at the antennae (Horneffer et al. 2004). Figure 1 shows the intensity maximum of the image-slices as a function of time ( $x$-axis) and curvature radius (different curves). The curves for the different curvature radii from $1 \mathrm{~km}$ to $10 \mathrm{~km}$ show that the intensity in the signal at $-1.8 \mu \mathrm{s}$ is increasing until the best fit is found, whereas the intensity of the background noise stays the same.

The intensity of the maximum source in each skymap increases steeply, when increasing the curvature radii from $1 \mathrm{~km}$ to about $3 \mathrm{~km}$, which is due to increasing coherence of the cosmicray air shower pulses in the individual antenna signals. For larger curvature radii, the intensity either keeps increasing asymptotically or a maximum is found, as in Fig. 2. For the plotted event the intensity peaks at $3 \mathrm{~km}$.

The intensity as a function of azimuth, elevation, and curvature radius (different curves) is shown in Fig. 3. The different

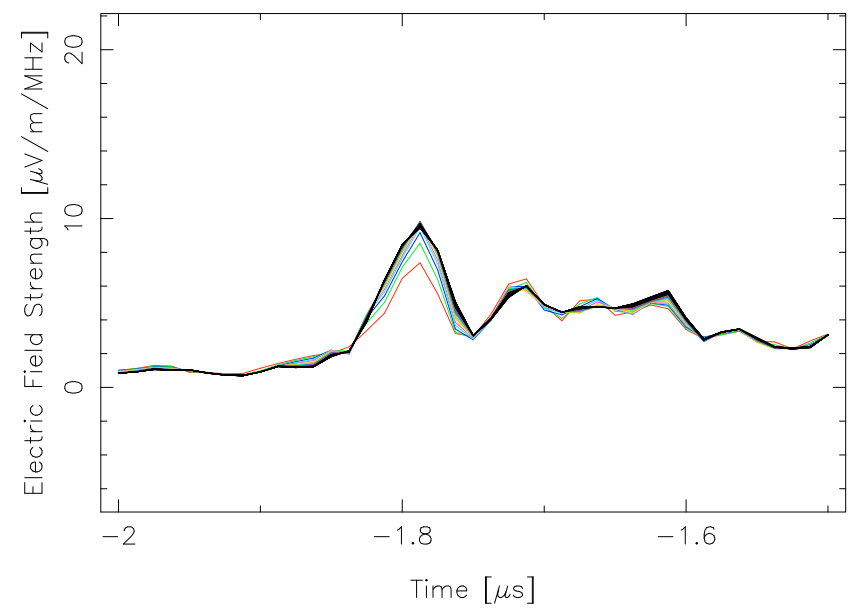

Fig. 1. Maximum electric field amplitude of the image as a function of time for different curvature radii. The amplitude of the pulse at $-1.8 \mu \mathrm{s}$ increases with beam-forming curvature radii (as shown for another event in Fig. 2).

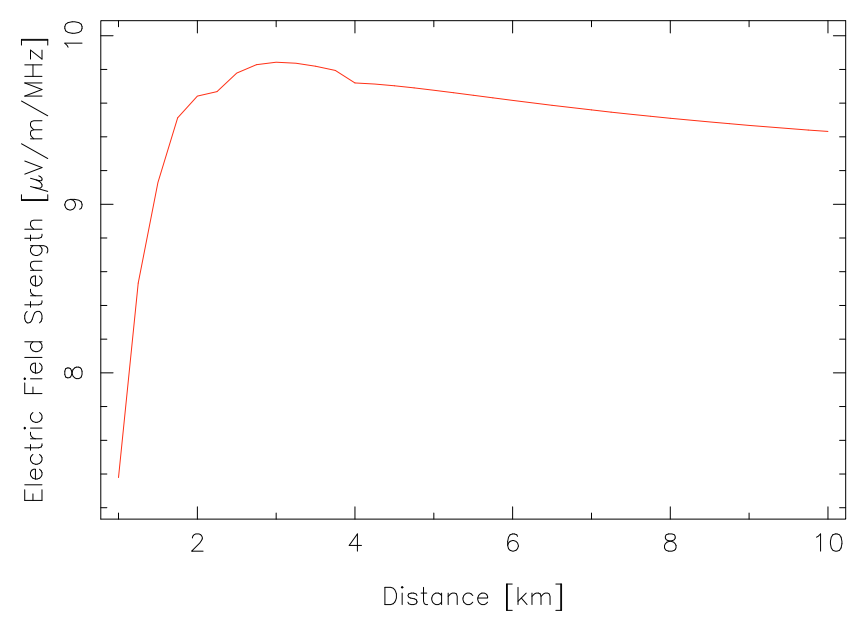

Fig. 2. Maximum electric field amplitude of the image as a function of beam-forming radius (distance from the observer to the beam focus) for an event with an estimated primary energy of $3 \times 10^{17} \mathrm{eV}$.

curves show with increasing curvature radius the increase in the intensity of the pulse, while the intensity of the side-lobes next to the pulse stay the same or are reduced in intensity.

In addition to the change in the intensity, in the plot with intensity versus elevation (bottom panel of Fig. 3), the position of the pulse-maximum shifts with increasing curvature radius to higher elevations by about two degrees. We attribute this to the offset between the phase center of the LOPES array and the shower core position as provided by KASCADE. The larger the distance between both, the more the position of the emission maximum changes, when varying the curvature radius for the beam-forming.

This beam-forming effect was tested by artificially introducing an extra delay by half a sample to all antenna data. This subsample shift is equivalent to a half-sample interpolation of the data, when correcting the time-axis by half a sample in the opposite shifting direction. The result of this method is displayed in Fig. 4, which is showing the skymaps for the unmodified and for the shifted data, respectively. It turns out, that the interpolation of the antenna data, from a sample time of $12.5 \mathrm{~ns}$ to $6.25 \mathrm{~ns}$, can further optimize the electric field amplitude of the air shower signal found in the beam. This is due to the sampling of the LOPES 

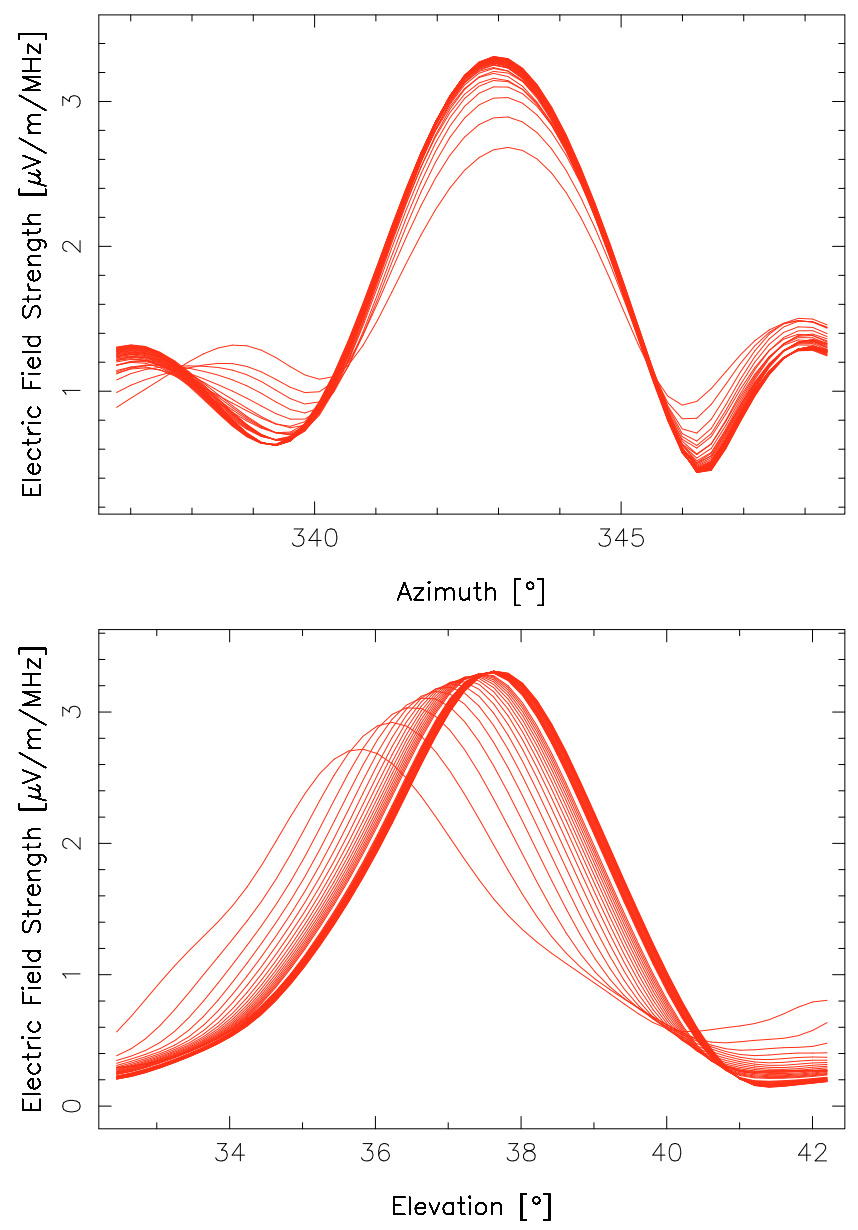

Fig. 3. Electric field amplitude as a function of azimuth (top) and elevation (bottom) at the time of maximum emission in the image for different curvature radii. The amplitude of the curves increases with beam-forming radii (as shown for another event in Fig. 2). The radius of curvature is increased by steps of $250 \mathrm{~m}$.

antennae signals in the second Nyquist-zone from $40 \mathrm{MHz}$ to $80 \mathrm{MHz}$ with a sample frequency of $80 \mathrm{MHz}$. Second Nyquistsampling preserves all signal information, which can be recovered by digital upsampling with, in our case, a factor of two.

We effectively did this by analyzing the interpolated data. In addition to an increase in the electric field amplitude, the pulse shifts in position, as observed when optimizing the curvature radius. Therefore, we conclude that the upsampling is necessary for the determination of the correct electric field amplitude. As expected, this will increase the accuracy of LOPES in field strength and direction estimate.

\section{Event selection}

The 664 pre-selected events (see Sect. 2) were processed as described above, including the half-sample shift. Out of these 664 events, 232 were selected having a minimum E-field peak SNR of 6 . The cut on the SNR of the events has been applied since for low SNR the measured noise from the KASCADE particle detectors dominates the air shower emission, which leads to a wrong fit when searching for the pulse profile in the time window of the 4D image.

The plot in Fig. 1 shows this window and the air shower pulse lies at $-1.8 \mu \mathrm{s}$, with increased curvature radius rising above the particle detector noise of KASCADE at $-1.75 \mu$ s to $-1.6 \mu \mathrm{s}$. For
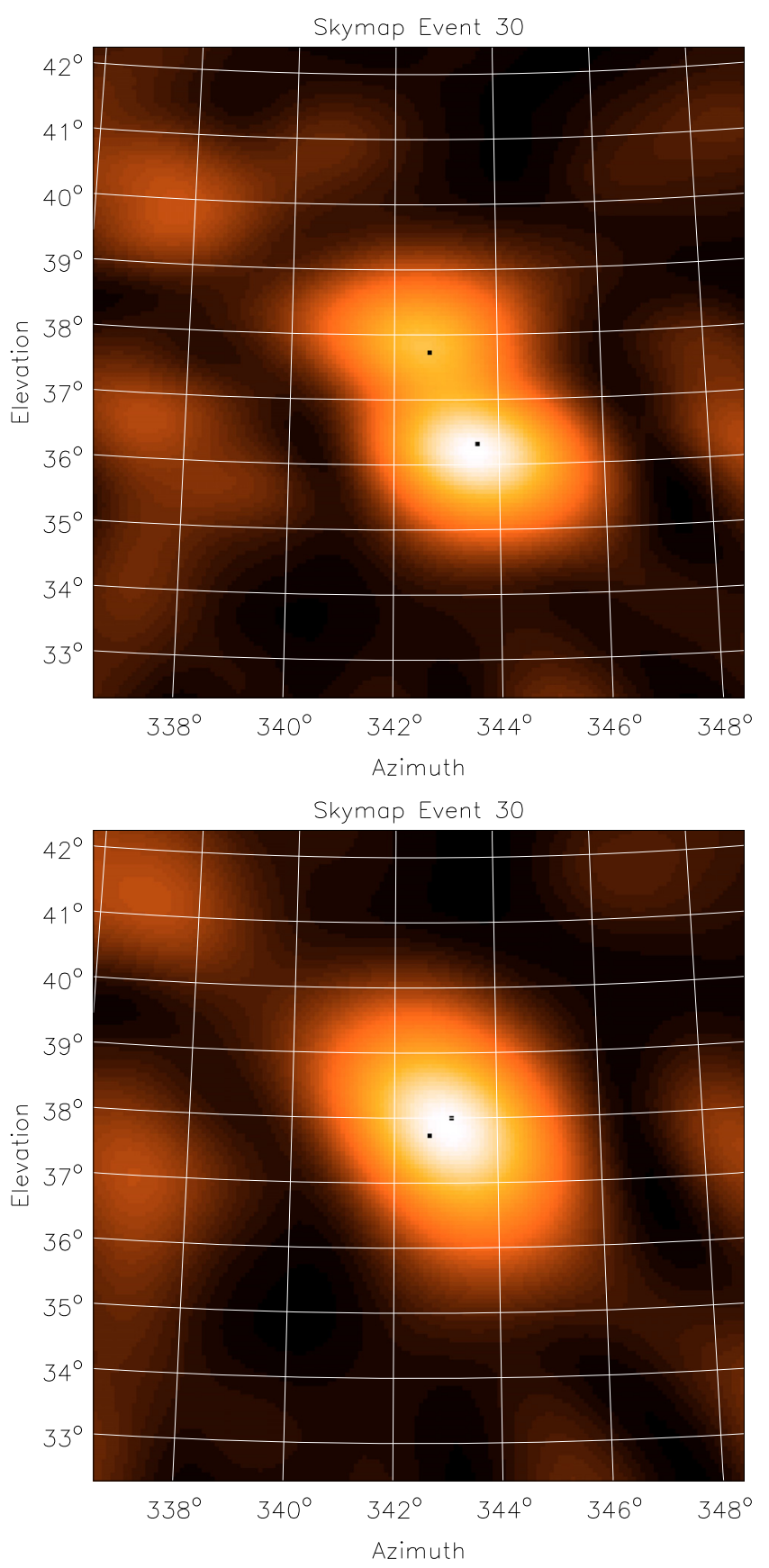

Fig. 4. Example of radio skymaps for one LOPES event with original sampling (top) and with a half-sample shift applied to all antennae signals before beam-forming (bottom). The split maximum in the top plot is not real and merges to one maximum with higher temporal resolution. The skymaps show the air shower radio emission in stereographic projection (STG, Calabretta \& Greisen 2002) with a black dot at the maximum. The second black dot in the center of the map and offset from the maximum indicates the direction provided by KASCADE.

events with a SNR below 6 the cosmic-ray radio pulse does not exceed the emission from KASCADE and the wrong signal is fitted. A third selection criterion on the coherence of the signal, obtained from the cross-correlation beam, and a fourth criterion on the elevation angle not to be smaller than $15^{\circ}$ reduced the number of the selected events further to 62 .

The cross-correlation beam is formed by correlating all paired combinations of the shifted antenna signals. The third selection criterion required the ratio of the electric field 


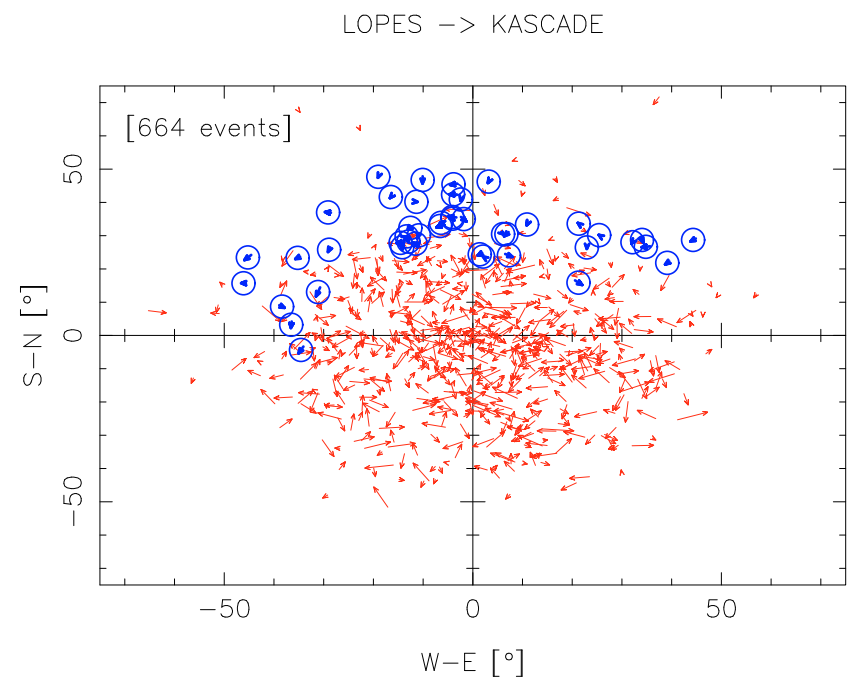

Fig. 5. This plot shows an arrow for each event connecting the position of LOPES and KASCADE projected onto the sky with the zenith in the origin. The azimuth angle rotates clock-wise starting from North pointing upwards. The thin arrows indicate all 664 pre-selected events and the thick encircled arrows highlight the 44 selected events.

amplitudes of the cross-correlation beam and the total power beam to be larger than 0.73 , which is one sigma above the average fraction of all events. This selection favors inclined events, since RFI from the KASCADE detectors is reduced, due to their reduced acceptance to larger zenith angles (see Fig. 5 and Petrovic et al. 2007). This way, events which consist of RFI but have a SNR above 6 are rejected. Such events were found to have small curvature radii, since most RFI is emitted locally. We note, that by this criterion also events were rejected from air showers which did not reach the required level of signal-coherence.

The rejection of RFI events can be tested by plotting a histogram of the distribution of the curvature radii. It can be seen in Fig. 6 that indeed all events which had an emission maximum at a curvature radius below $2000 \mathrm{~m}$ are not part of the filtered distribution, these events are considered as RFI events.

Among the 62 selected events, 17 events have an electric field strength of more than twice the expected field strength estimated from the muon number, geomagnetic angle, and shower axis distance (Horneffer et al. 2007). The measured electric field strength of the 62 selected events is plotted versus the directional offset in Fig. 7. In this plot, the 17 enhanced events are marked with squares and the 5 encircled events were confirmed to be recorded during thunderstorm activity with lightnings in the vicinity of LOPES. The error bars in the plot give the minimum uncertainty calculated with Eq. (1).

\section{Results and discussion}

The 5 encircled events of Fig. 7 are candidates for enhanced shower radio emission by electric fields in thunderstorm clouds (Buitink et al. 2007). These five thunderstorm events show large deviations in position, which suggests that the net force of the electric field in thunderstorm clouds can change the direction in which the emission is beamed. One of the 5 encircled events did not exceed the expected field strength by a factor of two, but had a strong offset in direction. The other 13 enhanced events could not be associated with thunderstorm activity, however, an effect by an increased geoelectric field in a cumulonimbus cloud without lightning is not excluded. Therefore, the enhanced events

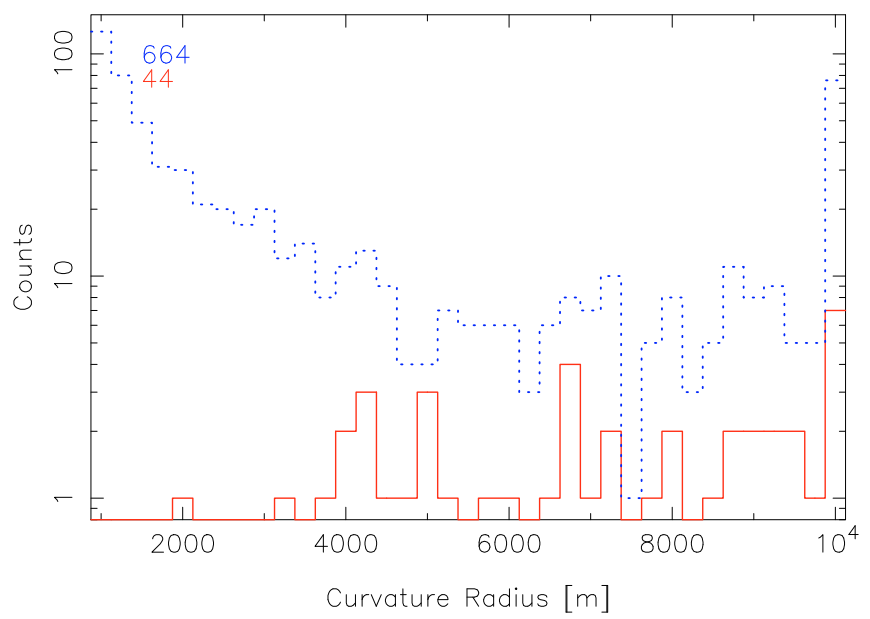

Fig. 6. Histogram of the number of events as a function of curvature radius. The upper (dotted-blue) distribution shows all 664 preselected events and the lower (solid-red) distribution shows the selected 44 events. The bin size is $250 \mathrm{~m}$ in curvature radius.

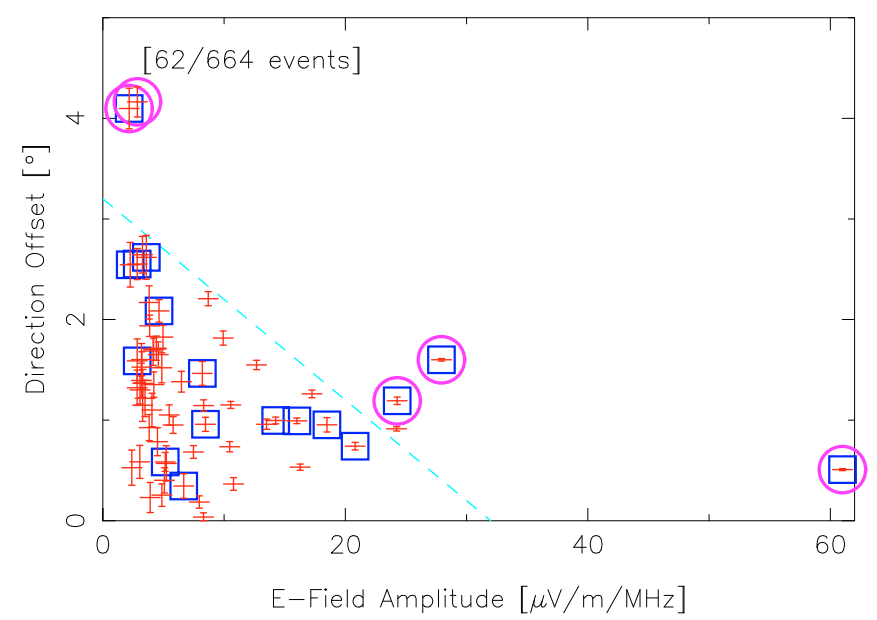

Fig. 7. Absolute angular separation between the LOPES and the KASCADE position as a function of electric field amplitude. The encircled events are possibly enhanced in field strength in thunderstorm clouds at the time of the observation. The events marked with squares have a measured field strength of more than twice the expected field strength estimated from the muon number, geomagnetic angle, and shower axis distance (Horneffer et al. 2007). The lower limit on the statistical uncertainty in the direction of each event is plotted as an error bar (according to Eq. (1)).

and the thunderstorm events were not taken into account for the following interpretation of the results. The remaining 44 events show an approximately linear decrease in directional offset with increasing signal strength.

\subsection{Curvature radius}

As mentioned in Sect. 3.3, the intensity detected in the pulses increases quickly with curvature radius. In fact, $90 \%$ of the maximum intensity is obtained for the selected 44 events at $(2600 \pm 800) \mathrm{m}$ and the curvature radii at the maxima are $(7000 \pm 2000) \mathrm{m}$ (one sigma statistical uncertainties). The statistical uncertainty for the radii at the maxima is large, because the change in the intensity at large curvature radii is small and cannot be determined more accurately with an array of the size 


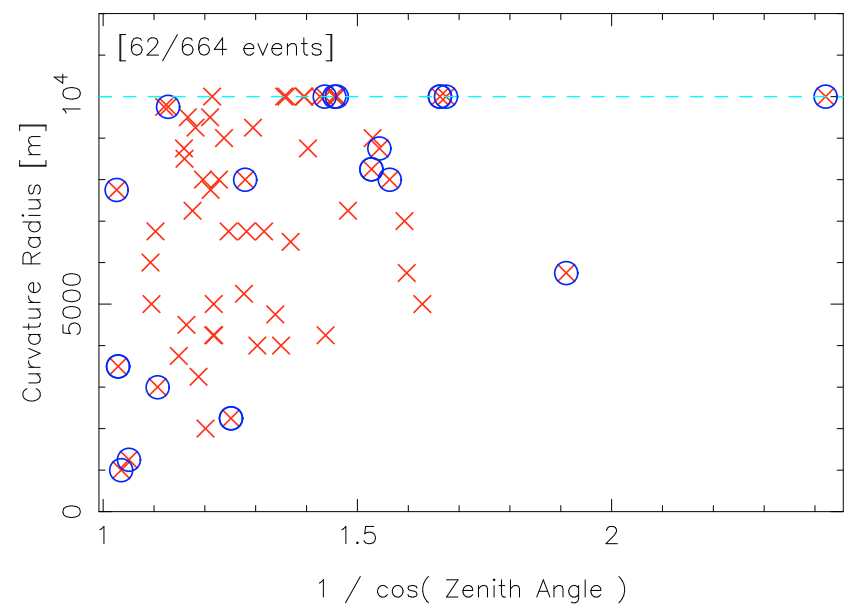

Fig. 8. Curvature radius as a function of the inverse cosine of the zenith angle for all 62 events. The enhanced and thunderstorm events are encircled in this plot. The dashed line at $10 \mathrm{~km}$ indicates the maximum curvature radius that was fitted to the wavefronts.

of LOPES. An approximation for the statistical uncertainty of the curvature radius can be obtained adapting Eq. (1):

$$
\left(\frac{\Delta \rho}{\rho}\right)_{\min }= \pm \frac{\rho}{D} \frac{\epsilon_{\mathrm{N}}}{\epsilon_{\mathrm{P}}} \frac{\lambda}{D \cos \theta} .
$$

Here $\Delta \rho$ is the uncertainty of the radius of curvature $\rho=7000 \mathrm{~m}$ and for $D=130 \mathrm{~m}, \lambda=5 \mathrm{~m}, \epsilon_{\mathrm{N}} / \epsilon_{\mathrm{P}}=1 / 16$, and $\theta=40^{\circ}$ results in $\pm 1400 \mathrm{~m}$, which is consistent with the above measured $\pm 2000 \mathrm{~m}$.

The histogram of the curvature radii in Fig. 6 shows that a few events accumulate at $10 \mathrm{~km}$ indicating that those events could be fitted with even larger curvature radii. However, on average, the intensity does not change significantly increasing the curvature radius over the last $2 \mathrm{~km}$, and therefore the wavefront is considered to be nearly planar. A tendency to larger curvature radii is observed for events coming from lower elevation angles, which travelled a longer path through the Earth's atmosphere (see Fig. 8).

Furthermore, we showed that the variation of the curvature radius can change the direction of the pulse in elevation. We found that the angular offset in elevation from the optimum position as a function of curvature radius is on average zero for the 44 selected events and therefore does not introduce a systematic effect. However, the spread must be considered as a statistical uncertainty of $\pm 0.3^{\circ}$ for the directions determined with LOPES.

\subsection{Upsampling}

Additionally, we showed in Sect. 3.3, that shifts in the directions occur when maximizing the intensity of the pulse by interpolating the antenna signals. The change in the positions introduced by the half-sample shifts amounts to $(0.7 \pm 0.9)^{\circ}$, for the 44 selected events. This result determines the improvement of the directional accuracy of LOPES. Effectively, the digitization step error was reduced, after the signal was upsampled.

The optimization of the beam-forming by the upsampling (half-sample shift) did not significantly increase the electric field amplitude of the selected events with $(1 \pm 3) \mu \mathrm{V} \mathrm{m}^{-1} \mathrm{MHz}^{-1}$ compared to the values determined with the standard analysis software (Bähren et al. 2006). However, it reduced the number of events with more than one source in the optimized skymap by $30 \%$. Thus, the intensity of the environmental background
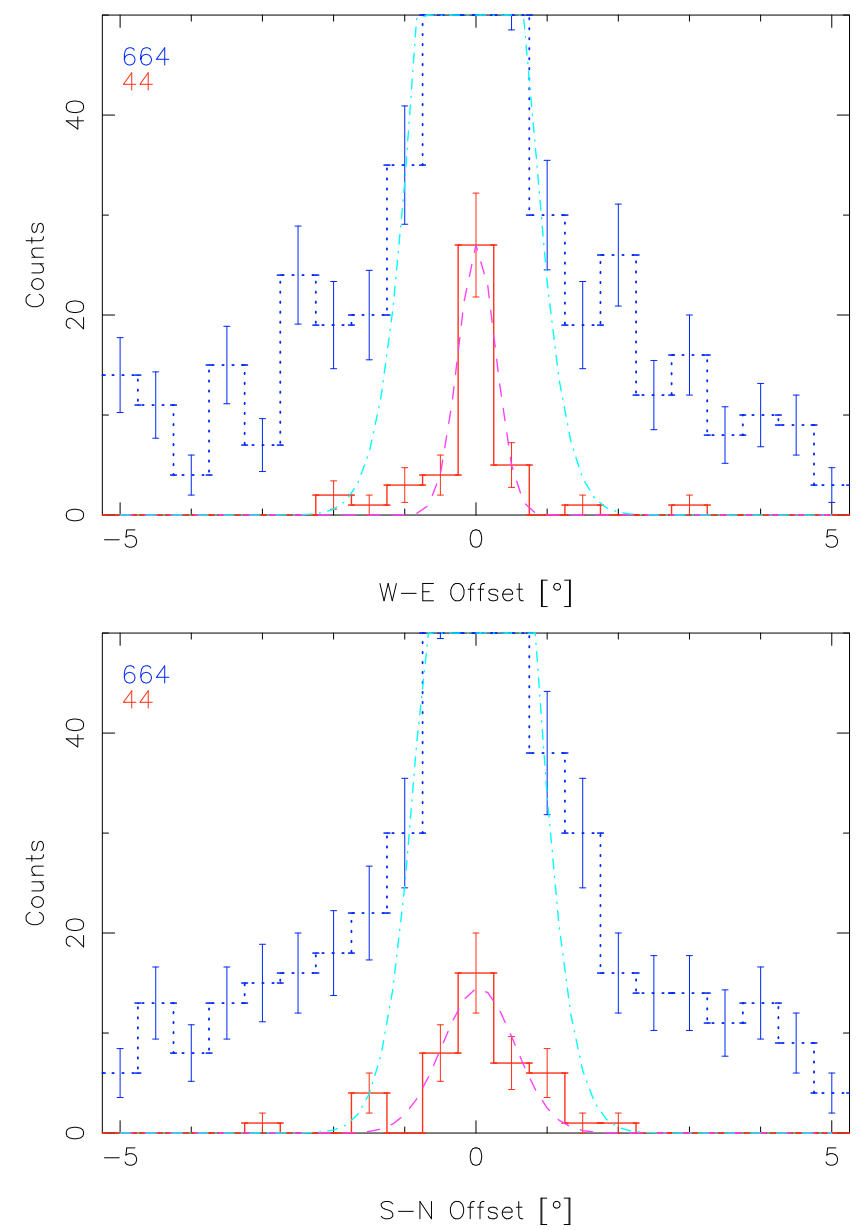

Fig. 9. Histograms of the angular offset in the air shower core position on the sky at the distance of the curvature radius of the air shower emission maximum, as found in the LOPES radio signal and as determined with the direction provided by KASCADE. The distribution in EastWest offset is plotted at the top and in North-South direction at the bottom. Each plot shows the distribution for all 664 events (dotted) and for the 44 selected events (solid), both fitted with a Gaussian (dash-dotted and dashed).

noise in the side-lobes of the event beams was reduced by the optimized beam-forming.

\subsection{Systematics}

Figure 5 shows arrows pointing from the position reconstructed by LOPES to the position reconstructed by KASCADE. The arrows for all events are isotropically distributed in the circular area that covers the sky. However, the arrows of the 44 events (selected on their E-field peak SNR) show a clear preference for coming from the North. Due to the inclination of the magnetic field of $65^{\circ}$ at the latitude of LOPES of $49^{\circ}$, the geomagnetic angles are larger to the North than to the South and therefore a larger signal is produced in the air shower by the geomagnetic effect.

The components of the arrows in the East-West direction and the North-South direction are plotted in Fig. 9. Both data sets follow a normal distribution with a full width at half maximum (FWHM) of $1.2^{\circ}\left(\chi_{\text {red }}^{2}=2.37\right)$ for the North-South direction and of $0.6^{\circ}\left(\chi_{\text {red }}^{2}=0.88\right)$ for the East-West direction. These values are consistent with the absolute offset of $(1.3 \pm 0.8)^{\circ}$ (see next section). Furthermore, both distributions have an average 


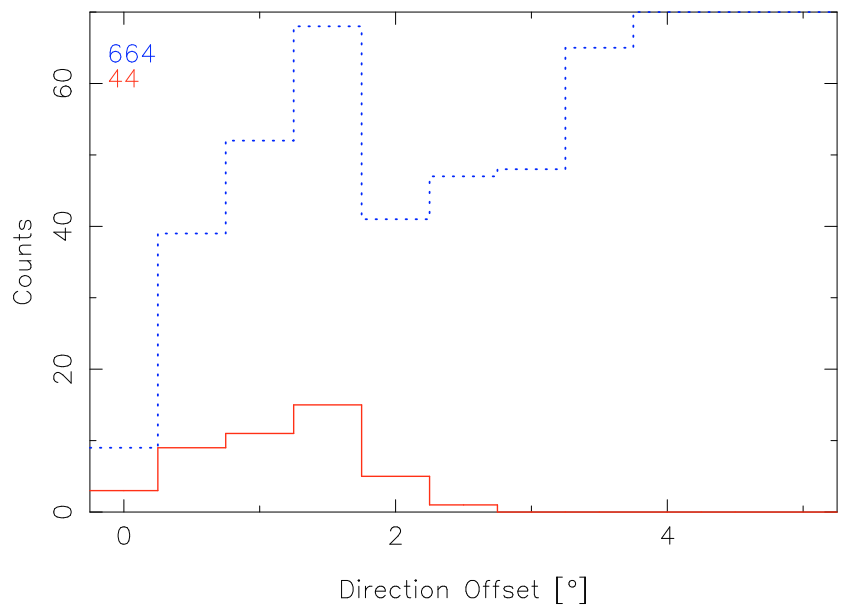

Fig. 10. Distribution of events as a function of directional offset between LOPES and KASCADE. The upper (dotted) distribution shows all 664 pre-selected events and the lower (solid) distribution shows the selected 44 events with the maximum at $(1.3 \pm 0.8)^{\circ}$. The bin size is $0.5^{\circ}$.

consistent with zero and therefore, no systematic offset is found in the directions determined by LOPES and KASCADE.

\subsection{Statistics}

The obtained offsets between the directions provided by KASCADE and the directions determined from the LOPES radio data for the 44 selected events result in $(1.3 \pm 0.8)^{\circ}$. The histogram of these offsets and of all 664 events peak at the same bin of $1.5^{\circ}$ suggesting that this is the sum of the average uncertainty for the direction determination of both experiments for the whole range of elevation angles. All events having larger offsets are dominated by increased noise from the particle detectors and they are not part of the selected 44 events (see Fig. 10).

The clear peaks in the curves for all 664 events of Figs. 9 and 10 indicate that the direction was successfully determined within $1.3^{\circ}$ for more than the 44 events. However, those events could also consist of RFI. The distinction between cosmic-ray events and RFI events could be improved by a better knowledge of the position and shape of the air shower pulse in time. This knowledge would allow a fit of the pulse in Fig. 1, which would enable the direction identification of events detected with less intensity than the corresponding KASCADE noise in the LOPES beam.

The 44 selected events have an average zenith angle of $40^{\circ}$ and the average electron number detected by KASCADE is $10^{6.8}$. The accuracy of KASCADE for an air shower with this electron number is $\pm 0.2^{\circ}$. This value is corrected for the reduced acceptance of the particle detectors by dividing with the sine of the zenith angle of $40^{\circ}$. Therefore, we conclude that the measured offsets are dominated by the accuracy of the direction determination with LOPES of about $\pm 1.1^{\circ}$.

This accuracy of LOPES lies above the statistical uncertainty determined with Eq. (1) of $\pm 0.2^{\circ}$, since the fit of a curved wavefront with a curvature radius $\rho$ introduces an additional uncertainty:

$\Delta \alpha_{\mathrm{tot}}= \pm \frac{1}{2} \frac{\epsilon_{\mathrm{N}}}{\epsilon_{\mathrm{P}}} \frac{\lambda}{D \cos \theta}+f(\rho)$.

\section{Conclusions}

The 4D mapping of radio emission is a powerful new tool for the study of radio sources within the Earth's atmosphere, in particular cosmic-ray air showers, because of the information in the third spatial dimension provided by the distance of the beamforming focus. We used an array with only 30 single dipoles and succeeded to map out the radio emission from air showers, to search for the position on the sky, and to fit the curvature of the detected wave front of the shower pulse. For this work, we used KASCADE as a reference. The mapping-tool can also be used for astrophysical imaging of fast pulses, since images can be calculated on sample time resolution. Furthermore, the third spatial dimension of the images allows to distinguish between the location of all kinds of transient events occurring inside and outside the Earth's atmosphere.

We found that the position of the emission maximum in the map, calculated in the beam-forming process, is sensitive to the array layout and the phase errors of the antenna electronics and therefore changes when beam-forming parameters such as the curvature radius or the filtered band are varied. We optimized the beam-forming by upsampling of the antenna signals, which strongly improved the accuracy of the direction determination.

We conclude that the angular resolution of LOPES is sufficient to localize the air shower axis and to maximize the received electric field amplitude. However, the accuracy for the direction determination is determined by the uncertainty of the curvature radius. An exact determination of the shape of the shower wavefront seems to be necessary to further improve the localization of the shower radio maximum in the beam-forming process. Furthermore, the directional accuracy could possibly be improved by larger baselines and by calculating every pixel of the searched skymap with the cross-correlation beam (sum of all antenna pair correlations) and by further suppressing the KASCADE noise. Also, more phase-stability of the electronics and a larger antenna layout optimized for beam-forming in all azimuthal directions is desirable.

We found that the best fit in the curvature radius increases for events coming from the horizon after passing through a larger atmospheric depth. Furthermore, we found an approximately linear decrease in the directional offsets between the LOPES positions and the KASCADE positions with increasing signal strength.

Furthermore, we found a few events with field strengths enhanced by more than twice the expected field estimated from the muon number, geomagnetic angle, and shower axis distance. Some of these events were associated with thunderstorms in the vicinity of the telescope, which can enhance the emission through strong electric fields in clouds. These events showed the largest offsets between the position measured with the radio antennae of LOPES and the particle detectors of KASCADE. This very important result suggests that the charged particles of air showers experience additional deflection by electric fields in thunderstorm clouds. Thus, we measured for the first time a geoelectric effect in addition to the geomagnetic effect.

The offsets between the directions measured with both instruments were analyzed for dependencies on the measured electric field strength, the curvature radius, the azimuth angle, the elevation angle and the geomagnetic field angle. Due to the geomagnetic effect and the geoelectric effect discussed here, one could expect a net directional offset between the muons, the electrons and the electromagnetic emission at the shower 
maximum. However, the analyzed sample of LOPES events did not show any significant systematic effect for the mentioned dependencies.

This result adds another strong argument for the usage of the radio detection technique for the study of the arrival directions of high-energy cosmic rays. An interesting next step will be to investigate with simulations how the distance of the beam focus (curvature radius) relates to the maximum of the particle shower.

Acknowledgements. Andreas Nigl gratefully acknowledges a grant from ASTRON which made this work possible. LOPES was supported by the German Federal Ministry of Education and Research. The KASCADE-Grande experiment is supported by the German Federal Ministry of Education and Research, the MIUR and INAF of Italy, the Polish Ministry of Science and Higher Education and the Romanian Ministry of Education and Research.

\section{References}

Allan, H. R., \& Jones, J. K. 1966, Nature, 212, 129

Antoni, T., Apel, W. D., Badea, F., et al. 2003, Nucl. Instr. Meth. Phys. Res. Sect. A, 513, 490

Ardouin, D., Bellétoile, A., Charrier, D., et al. 2005, in SF2A-2005: Semaine de l'Astrophysique Francaise, ed. F. Casoli, T. Contini, J. M. Hameury, \& L. Pagani, 495

Auger, P., Ehrenfest, P., Maze, R., Daudin, J., \& Fréon, R. A. 1939, Rev. Mod. Phys., 11, 288

Auger Collaboration: J. Abraham et al. 2007, Science, 318, 938

Bähren, L., Falcke, H., Horneffer, A., Lafebre, S., \& Nigl, A. 2006, The LOPESTools Software Package, http://usg. lofar.org/doku.php

Buitink, S., Apel, W., Asch, T., et al. 2007, A\&A

Calabretta, M. R., \& Greisen, E. W. 2002, A\&A, 395, 1077

Falcke, H., Apel, W. D., Badea, A. F., et al. 2005, Nature, 435, 313

Falcke, H., \& Gorham, P. 2003, Astropart. Phys., 19, 477

Horneffer, A., Apel, W. D., Arteaga, J. C., et al. 2007, in Proc. Int. Cosmic Ray Conf. (ICRC)
Horneffer, A., Apel, W. D., Badea, A. F., et al. 2004, in Gravitational Wave and Particle Astrophysics Detectors, ed. J. Hough, \& G. H. Sanders, Proc. SPIE, 5500,129

Huege, T., \& Falcke, H. 2005, Astropart. Phys., 24, 116

Jelley, J. V., Fruin, J. H., Porter, N. A., et al. 1965, Nature, 205, 327

Mayer, H. J. 1992, Nucl. Instr. Meth. Phys. Res., Sect. A, 317, 339

Mayer, H. J. 1993, Nucl. Instr. Meth. Phys. Res., Sect. A, 330, 254

Nigl, A., Apel, W. D., Arteaga, J. C., et al. 2008, A\&A, in press

Petrovic, J., Apel, W. D., Asch, T., et al. 2007, A\&A, 462, 389

Shannon, P. 1996, in Astronomical Data Analysis Software and Systems V, ed. G. H. Jacoby, \& J. Barnes, ASP Conf. Ser., 101, 319

Taylor, G. B., Carilli, C. L., \& Perley, R. A. 1999, Synthesis Imaging in Radion Astronomy II, ASP Conf. Ser., 180

1 Department of Astrophysics, IMAPP, Radboud University Nijmegen, PO Box 9010, 6500 GL Nijmegen, The Netherlands e-mail: anigl@astro.ru.nl

2 Institut für Kernphysik, Forschungszentrum Karlsruhe, 76021 Karlsruhe, Germany

3 Institut für Experimentelle Kernphysik, Universität Karlsruhe (TH), 76021 Karlsruhe, Germany

4 Institut für Prozessverarb. und Elektr., Forschungszentrum Karlsruhe, 76021 Karlsruhe, Germany

5 Fachbereich Physik, Universität Wuppertal, 42097 Wuppertal, Germany

6 ASTRON, 7990 AA Dwingeloo, The Netherlands

7 Dipartimento di Fisica Generale dell'Università, 10125 Torino, Italy

8 Max-Planck-Institut für Radioastronomie, 53010 Bonn, Germany

9 National Institute of Physics and Nuclear Engineering, 7690 Bucharest, Romania

10 Fachbereich Physik, Universität Siegen, 57068 Siegen, Germany

11 Istituto di Fisica dello Spazio Interplanetario, INAF, 10133 Torino, Italy

12 Soltan Institute for Nuclear Studies, 90950 Lodz, Poland

13 Physics Department, Bucharest University, Bucharest-Magurele, PO Box MG-11, 077125, Romania 